\title{
PRATİKTE KULLANILAN BAZI KAYAN KİPLİ KONTROL TEKNİKLERİNİN TEORİK VE DENEYSEL İNCELENMESİ
}

\author{
Mehmet Buğrahan KALAYCI, İlhami YİĞİT \\ Bozok Üniversitesi Mühendislik Mimarlık Fakültesi, YOZGAT \\ bugrahan.kalayci@bozok.edu.tr, ilhami.yigit@bozok.edu.tr
}

(Geliş/Received: 12.06.2014; Kabul/Accepted: 26.02.2015 )

\section{ÖZET}

Kayan kipli kontrol, uygun şartlar yerine geldiğinde, dış bozucular ve belirsizliklere rağmen istenilen dinamik davranışı sağlayabilen gürbüz bir kontrol yöntemidir. Kayan kipli kontrol yöntemi, uygulama basitliği ve gürbüzlüğünden dolayı, son yıllarda gözde hale gelmiş, çok sayıda kayan kipli kontrol tekniği geliştirilmiş, mühendislik uygulamalarda yerini almıştır. Ancak, açık literatürde bu kontrol tekniklerinin çeşitli gerçek sistemler üzerinde aynı anda uygulandığı kapsamlı bir çalışma bulunmadığı görülmüştür. Bu çalışmada, pratikte kullanılan bazı kayan kipli kontrol teknikleri çeşitli gerçek sistemler üzerinde teorik ve deneysel olarak incelenmiştir. Bu amaçla, tek giriş tek çıkışlı doğru akım motoru tahrikli doğrusal bilyalı kızak sistemi, doğrusal olmayan basit ters sarkaç ve tek giriş çok çıkışlı dolaylı tahrikli doğrusal olmayan arabalı ters sarkaç sistemi olmak üzere üç farklı gerçek sistem kullanılmıştır. Bu sistemlerin kontrolleri, hem teorik hem de deneysel olarak tatmin edici düzeyde gerçekleştirilmiş, elde edilen çok sayıda simülasyon ve deney sonuçları değerlendirilerek karşılaştırmalar yapılmış, çeşitli sonuçlar çıkarılmış, ileriye dönük çalışmalar için önerilerde bulunulmuştur.

Anahtar Kelimeler: kayan kipli kontrol, doğrusal olmayan sistem, ters sarkaç, modelleme, simülasyon, deney

\section{THEORETICAL AND EXPERIMENTAL INVESTIGATION OF SOME SLIDING MODE CONTROL TECHNIQUES USED IN PRACTICE}

\begin{abstract}
Sliding mode control is a robust control technique which is able to provide a desired dynamic behaviour in spite of disturbances and uncertainties under matching conditions. In recent years, because of simplicity and robustness, sliding mode control has become popular and various sliding mode control techniques have been developed and taken place in engineering practices. However, it is found that a comprehensive study dealing with the application of these control techniques on various real systems is not available in open literature. In this study, some commonly used sliding mode control techniques are investigated theoretically, and experimentally applying on various real systems. For this purpose, three different types of real systems are used: a single input single output direct current motor driven linear recirculating ball bearing system; a nonlinear simple inverted pendulum; and a single input multiple output underactuated nonlinear cart type inverted pendulum. Control of the systems with various control techniques are implemented satisfactorily both theoretically and experimentally. Results of simulations and experiments are compared with each other, and various inferences are made and recommendations for further studies are presented.
\end{abstract}

Keywords: Sliding mode control, nonlinear system, inverted pendulum, modelling, simulation, experiment

\section{GIRIŞ (INTRODUCTION)}

Kayan kipli kontrol, uygun şartlar yerine geldiğinde [1], sistemdeki belirsizliklere, parametre değişimlerine ve bozuculara rağmen istenilen dinamik davranışı sağlayabilen son derecede gürbüz bir kontrol tekniğidir. 1950'lerde ilk defa Emelyanov tarafından ortaya atılan teknik, gelişen hızlı anahtarlama teknolojisi ve 1977 yılında ilk kez Vadim Utkin'in İngilizce olarak yayınlanan makalesi ile ciddi anlamda gündeme gelmiş [2], günümüze kadar da gittikçe artan bir ivme ile kullanım alanı genişlemiştir. Kayan kipli kontrol Vadim Utkin tarafından şu şekilde tanımlanmaktadır: "Kayan kipli kontrol, sürekli kontrol fonksiyonlarıly oluşmuş uygun anahtarlama mantı̆̆ 
sistemler kümesinden oluşur. Kontrol tekniğinin sistemi durum uzayında belli bir yüzey üzerinde süreksizliğe maruz bıraktığı kabul edilir.“ " [3].

Gerçek dinamik sistemlerde, modelleme hataları, istenmeyen sıcaklık, basınç veya voltaj değişimleri, titreşim ve bozucu girişler gibi çeşitli çevresel faktörlerden kaynaklanan belirsizliklerden kaçınmak imkânsızdır. Endüstride kullanılan klasik doğrusal kontrolcüler (PID kontrol gibi) genellikle parametre değişimlerine, modellenmemiş dinamiklere ve bozucu girişlere karşı tatminkâr sonuçlar verememektedir. $\mathrm{Bu}$ nedenle, bozucuların ve belirsizliklerin sistem cevabı üzerindeki etkisini yok edebilen kayan kipli kontrol tekniği tercih edilmektedir [4].

Yapılan literatür araştırmalarında, çok sayıda kayan kipli kontrol tekniğinin farklı türden gerçek sistemler üzerinde aynı anda uygulandığ 1 teorik ve deneysel bir inceleme çalışmasının bulunmadığını göstermiştir [513]. Literatürde, çoğunlukla bir tekniğin özellikleri ortaya konulup klasik bir veya iki teknik ile karşılaştırması yapılmış, ya yalnızca teorik çalışma ile yetinilmiş yada bir deney düzeneği üzerinde uygulamalı çalışılmıştır. SUSPERREGUI ve ark., doğru akım (dc) servo motor açısal referans takibi için iki ayrı birinci mertebeden kayan kipli kontrol yaklaşımı uygulamışlardır. Birinci yaklaşım Slotine'in genel yaklaşımı ve ikincisi de Utkin'in yaklaşımıdır. Kontrol için tam mertebe Luenberger gözlemleyici tasarlamışlardır. Simülasyon ve deneysel sonuçlarla her iki yaklaşımın da uygunluğunu ve parametre değişimlerine duyarsızlığını belirlemişlerdir [5]. EKER, Elektromekanik bir sistemin hız kontrolü için PID kayma yüzeyi kullanarak çalışmalar yapmıştır. Kayan kipli kontrol'ün tırlama etkisini gidermek için hiperbolik fonksiyon kullanmıştır. Deneysel çalışmalar PID kontrol ile kıyaslamıştır ve deneysel sonuçlar, önerilen kayan kipli kontrolcünün bozuculara ve belirsizliklere rağmen gürbüz olduğu doğrulamışlardır [6]. BRANDTSTÄDTER ve BUSS dc motor tahrikli ters sarkaç sisteminde, elektromekanik sistemler için önerdikleri kayan kipli kontrolü uygulamışlardır. Güç tüketimini düşüren, gürbüzlük sağlayan, uygulamada basitlik sağlayan ve daha iyi performans sağlayan yeni kontrol yaklaşımı önermiştir. Önerilen yaklaşımın performansı nümerik simülasyon ve deneyler ile doğrulamışlardır [7]. TAEDONG ve KIHEON doğrusal konum kontrolünde, kök yerleştirme ile belirlenen kayma yüzeyi ile kontrolün performansını deneysel olarak analiz etmişlerdir. Yüksek kontrol aktivesini elimine etmek için işaret fonksiyonu yerine sigmoid-benzeri fonksiyon kullanmışlardır [8]. NAFA ve ark. bir sınıf dolaylı tahrikli sistem için düzenlenmiş kayan kipli kontrol önermişlerdir. 3 serbestlik derecesi olan dolaylı tahrikli mekanik sistem kontrol edilmiştir. Bu sistem 3 farklı alt sistem içermektedir ve her bir sistem için yüzey önerilmiştir. Yaptıkları arabalı çift ters sarkaç simülasyonu, önerilen metodun doğruluğunu göstermiştir [9]. RAO ve ark. bir dönel ters sarkaç sistemini, dc motor dinamiğini de modelleyerek, kontrol sinyalini motor akımı olarak seçerek kayan kipli kontrolünü gerçekleştirmişlerdir. Doğrusal olmayan bu sistemin davranışı, doyma fonksiyonu ile kontrol edilerek çalışmışlardır. Simülasyon ile kontrol sinyalinin akım seçildiği durumda çıkışların performanslarını göstermişlerdir [10]. SHIN ve ark. çalışmalarında, belirsizlikler içeren bir sınıf dolaylı tahrikli sitsem için bulanık mantık ile uyarlamalı ayarlama yapılan ayrıştırılmış kayan kipli kontrol tasarlamışlardır. $\mathrm{Bu}$ yöntem ile sistem alt sistemlere ayrıştırılır ve her bir alt sistemin farklı hedefleri olan kayma yüzeylerini tanımlamışlardır. Simülasyon sonuçları ile önerdikleri yaklaşımın doğruluğunu göstermişlerdir [11]. QIAN ve ark. çalışmalarında, seri çift ters sarkaç siteminin kontrolü için hiyerarşik kayan kipli kontrol yaklaşımı önermişlerdir. Sistemi alt sistemlere ayırmışlar, her bir alt sistem için yüzey tanımlamışlar ve her bir yüzey için kontrol kuralı oluşturmuşlardır. Bütün yüzeylerin asimptotik kararlığını garanti almışlardır. Yaptıkları simülasyonlar sonucu önerdikleri yöntemin geçerliliğini göstermişlerdir [12]. HAO ve ark. çalışmalarında, belirlisizlikler içeren bir sınıf dolaylı tahrikli kayan kipli kontrol metodu tasarlamışlardır. Dolaylı tahrikli sistemler için 2n olan durum değişkenlerine karşılık kontrolcü için 2n-1 yüzey tanımlamışlardır. Bütün yüzeylerin kararlılığını kanıtlamışlardır ve simülasyonlar sonucu önerdikleri yöntemin geçerliliğini göstermişlerdir [13].

Yukarıda özetlenen çalışmalardan da görüldüğü gibi, sistemini kayan kipli kontrol ile kontrol etmek isteyen bir kontrol mühendisinin hangi tür SMC tekniğinin sistemine daha uygun olacağını belirleyebilmesi için kapsamlı bir karşılaştırmalı inceleme çalıșması bulunmamaktadır. Buradan, kayan kipli kontrol tekniklerinin kapsamlı bir şekilde teorik ve deneysel olarak incelenmesi çalışmasının faydalı olacağı görülmüştür.

$\mathrm{Bu}$ çalışmada, pratikte kullanılan bazı kayan kipli kontrol teknikleri çeşitli sistemler üzerinde teorik ve deneysel olarak incelenmiştir. $\mathrm{Bu}$ tekniklerin incelenmesi amacıyla, tek giriş tek çıkışılı bir sistem olan doğru akım motoru tahrikli araba sistemi, direk tahrikli basit ters sarkacın yanı sıra, tek giriş çok çıkışlı bir sistem olan dolaylı tahrikli arabalı ters sarkaç sistemi olmak üzere üç farklı gerçek sistem üzerinde çalışmalar yapılmıştır.

Ters sarkaç sistemleri, kontrol sistemlerinin başarımının sınanmasında sıklıkla kullanılan doğrusal olmayan dolaylı tahrikli sistemler olması nedeniyle tercih edilmiştir. Sonuç olarak, çok sayıda kayan kipli kontrol tekniği teorik incelemeler ve simülasyon çalışmalarını müteakip çeşitli gerçek sistemler üzerinde çok sayıda deney ile gözlemlenmiş, uygulama şekilleri ve performansları hakkında çeşitli çıkarımlarda bulunulmuştur. 


\section{CALIŞMADA KULLANILAN KONTROL TEKNIKLERI (CONTROL TECHNIQUES USED IN THE STUDY)}

Bu çalışmada kullanılan kontrol teknikleri, Slotine'in yaklaşımı, integral kayan kipli kontrol ve Utkin'in yaklaşımı; dolaylı tahrikli ve tek giriş çok çıkışlı bir sistem olan ters sarkaç sistemine ise Utkin'in yaklaşımı, hiyerarşik kayan kipli kontrol ve kaskat kayan kipli kontrol uygulanmıştır. Klasik PID kontrol ile karşılaştırma yapılmış, yapıları benzer olan kayan kipli kontroller kendi aralarında karşılaştırılmıştır.

\subsection{PID Kontrol (PID Control)}

Hatanın mevcut, geçmiş ve gelecek durumunu dikkate alabilen PID kontrol, yapısının basitliği, uygulanabilirliğinin yüksek olması ve yeterli gürbüzlüğü sağlaması nedeniyle pek çok endüstriyel uygulamada tercih edilmektedir. Standart PID kontrol için kontrol sinyali, çoğunlukla,

$u_{p i d}=K_{p}\left(e(t)+\frac{1}{T_{i}} \int_{0}^{t} e(t) d t+T_{d} \frac{d e}{d t}\right)$

şeklinde ifade edilir[14]. Burada $K_{p}$ oransal, $T_{i}$ integral ve $T_{d}$ türevsel kazançlardır.

\subsection{Kayan Kipli Kontrol (Sliding Mode Control)}

Kayan kipli kontrol, uygun şartlar yerine geldiğinde, sistemdeki belirsizliklere, parametre değişimlerine ve bozuculara rağmen istenilen dinamik davranışı sağlayabilen son derecede gürbüz bir kontrol tekniğidir. 1950'lerde ilk defa Emelyanov tarafindan ortaya atılan teknik, gelişen hızlı anahtarlama teknolojisi ve 1977 yllında ilk kez Vadim Utkin'in İngilizce makalesi ile ciddi anlamda gündeme gelmiş [2], günümüze kadar da gittikçe artan bir ivme ile kullanım alanı genişlemiştir. Kayan kipli kontrolün temeli gürbüz kontrol olarak bilinen "değişken yapılı kontrol"e dayanmaktadır. Kayan kipli kontrol (KKK) yönteminde, sonsuz anahtarlamalı geribesleme kontrolü kullanılarak, doğrusal veya doğrusal olmayan sistem, durum uzayında tanımlanan yüzey üzerine çekilir ve yüzey üzerinde tutulur. $\mathrm{Bu}$ yüzey, durum değişkenlerinden oluşur, arzu edilen sistem cevabı şekline göre seçilir ve 'kayma yüzeyi' tanımlanır. Sistem kayma yüzeyine bir kez ulaştığ zaman, davranışı, kontrol edilen sistem (plant) parametre değişimlerinden ve bozucu etkilerden bağımsız hale gelir (değişmezlik koşulu).

Kayan kipli kontrolde, süreksiz bir yapı söz konusu olup anahtarlamalı kontrol sinyalinden ve diğer etkenlerden dolayı yüksek frekansta zigzag hareketi "tırlama" (chattering) meydana gelir. Tirlama, titreşim oluşturan, sistemin ömrünü kısaltan ve gereksiz enerji tüketimine neden olan olumsuz bir etkidir. Tırlamayı azaltmak veya ortadan kaldırmak için yüzeyin yakın bölgesinde sert geçişleri olan "sign" fonksiyonu yerine "sat " doyma fonksiyonu veya "tanh" sigmoid fonksiyonu gibi "yumuşatma fonksiyonları" kullanılabilir.

Kayan kipli kontrol teorisinde, kontrol dinamiğinin iki ardışık kipi vardır; birincisi ulaşma kipi ve ikincisi kayma kipidir [2,4,15].

Kayan kipli kontrol tasarımı iki aşamadan oluşur. İlk aşaması kayma yüzeyi ikinci aşaması kontrolcü tasarlanmasıdır. Kayma yüzeyi, arzu edilen kapalı çevrim sistem cevabı şekline göre kök yerleştirme metoduyla durum uzayında tasarlanır. İkinci aşamada ise kontrol sinyali Lyapunov kararlılık teoremi ile elde edilir. Lyapunov kayma koşulu sistemi yüzeye ulaşmaya zorlar ve yüzey üzerinde tutar. Sistemin kararlılığı seçtiğimiz kararlı yüzey ve kayma koşulu (Lyapunov kararlılık teoremi) ile sağlanmış olur [4]. Ulaşma kipinde, kontrol dinamiği sistem parametrelerine bağlıdır; kayma kipinde ise sistemin dinamiği tasarlanan yüzey parametrelerince belirlenir, kayan kipli kontrolün gürbüzlük özelliği kayma kipinde geçerli olur.

2.2.1. Kök Yerleştirme Yöntemi ile Kayma Yüzeyinin Belirlenmesi (Determination of Sliding Surface by using Pole Placement Method)

Doğrusal bir sistemi aşağıdaki gibi tanımlanabilir.

$\dot{x}=\boldsymbol{A x}+\boldsymbol{B u} \quad \boldsymbol{x} \in \mathcal{R}^{n}, u \in \mathcal{R}^{1}$

Sistem $\mathrm{T}$ dönüşüm matrisiyle "kontrol edilebilir kanonik form"a dönüştürülebilir. Ty $=x$ dönüşümü gerçekleştirilerek, sistemin kayma kipinde davranışı $[16,17]$,

$\dot{\boldsymbol{y}}=T^{-1} \dot{\boldsymbol{x}}$

$\dot{\boldsymbol{y}}=T^{-1}[\boldsymbol{A x}+\boldsymbol{b u}]$

$\dot{y}=T^{-1} A T y+T^{-1} b u$

bulunur. Burada $T$ matrisi sistemin "kontrol edilebilirlik matrisi"nden elde edilmektedir [16] ve

$T=\left(b, A b, \ldots, A^{n-1} b\right)\left[\begin{array}{ccccc}a_{2} a_{3} & \cdots & a_{n} & 1 \\ a_{3} a_{4} & \cdots & 1 & 0 \\ \cdots \cdots \cdots \cdots \cdots & \cdots & \cdots & \cdots \\ a_{n} & 1 & \cdots & 0 & 0 \\ 1 & 0 & \cdots & 0 & 0\end{array}\right]$

olarak tanımlanır. Ty $=x$ koordinat dönüşümünden sonra sistemin durum denklemi,

$\dot{\boldsymbol{y}}_{1}=\boldsymbol{A}_{11} y_{1}+A_{12} y_{2}$

$\dot{y}_{2}=A_{21} y_{1}+A_{22} y_{2}+u$

formuna dönüşür. Burada $\boldsymbol{y}_{\mathbf{1}} \in \mathcal{R}^{(n-m)}, y_{2} \in \mathcal{R}^{m}$ ve $A_{i j}, i, j=1,2$. , sabit matrislerdir. Kayma yüzeyi,

$s=y_{2}+C y_{1}=0$

olarak seçilirse sistemin s yüzeyindeki davranışı, 
$y_{2}=-C y_{1}$

ifadesiyle kendini gösterir. Kayma kipi davranışı,

$\dot{y}_{1}=\left(A_{11}-A_{12} C\right) y_{1}$

denklemiyle belirlenir. Denklem 7 ile ifade edilen kayma yüzeyi durum uzayında,

$s(x)=\boldsymbol{C}_{\boldsymbol{D}} \boldsymbol{x}$

$\dot{s}(x)=\boldsymbol{C}_{\boldsymbol{D}} \dot{\boldsymbol{x}}=C_{D}\left(\boldsymbol{A} \boldsymbol{x}+\boldsymbol{b} u_{e q}\right)=0$

$u_{e q}=-\left(\boldsymbol{C}_{\boldsymbol{D}} \boldsymbol{b}\right)^{-1} \boldsymbol{A} \boldsymbol{x}$

olarak bulunur. Toplam kontrol sinyali,

$u=-\left(\boldsymbol{C}_{\boldsymbol{D}} \boldsymbol{b}\right)^{-1} \boldsymbol{A} \boldsymbol{x}-k \operatorname{sign}(s)$

olarak elde edilir. Böylelikle sistemimizin modelinden yararlanılarak, arzu edilen kapalı çevrim kontrollü sistem davranış şekli elde edilmiş olur. Lyapunov kararlılık teorisine göre sonlu zamanda erişimi için[21], $s \dot{s}<-\mu|s|<0$ kriteri dikkate alınarak, toplam kontrol sinyali,

$u=-\left(\boldsymbol{C}_{\boldsymbol{D}} \boldsymbol{b}\right)^{-1} \boldsymbol{A} \boldsymbol{x}-\mu \operatorname{sign}(s) \cong u_{e q}+u_{s w}$

olarak elde edilmiş olur.

2.2.2. Referans Yörünge İzleme için Kayan Kipli Kontrol (Sliding Mode Control for Reference Trajectory Tracking Control)

Doğrusal olmayan tek girişli tek çıkışlı bir sistem bir çok durumda aşağıdaki gibi tanımlanabilir.

$\boldsymbol{x}^{(n)}=\boldsymbol{A}(\boldsymbol{x})+\boldsymbol{B}(\boldsymbol{x}) u$

Referans giriş durum vektörü $\boldsymbol{x}_{r}=\left[x_{r}, \dot{x}_{r}, \ldots, x_{r}^{(n-1)}\right]^{\mathrm{T}}$ şeklinde, sistemin çıkışı $x$ için hata $e=x-x_{r}$ ve hata vektörü $\boldsymbol{e}=$ $\left[e, \dot{e}, \ldots, e^{(n-1)}\right]^{\mathrm{T}} \quad$ şeklinde tanımlanmış olsun. Zamanla değişen kayma yüzeyi aşağıdaki ifade uyarınca tasarlanabilir [18].

$s(x, t)=\left(\frac{d}{d t}+k\right)^{n-1} e$

Sistemi zamanla değişen kayma yüzeyine ulaştıracak kontrol sinyali $u$ aşağıdaki gibi $\mu$ erişilebilirlik kriterinden elde edilebilir.

$\frac{d}{d t} s^{2}<-\mu|s|<0$

Burada, $\eta>0$ 'dır. Denklem 17, sistemi yüzeye ulaşmaya zorlar ve yüzeye ulaştığında da yüzey üzerinde tutar. Kontrol yöntemi ile ilgili detaylı çıkarımlar Kaynak 18 'de yer almaktadır.

\subsubsection{Integral Kayan Kipli Kontrol (Integral Sliding Mode Control)}

Kayma yüzeyi denklemine integral terimi eklenerek benzer sonuçlar elde edilebilir [9].

$s(t)=\left(k+\frac{d}{d t}\right)^{n-1} e(t)+k_{i} \int_{0}^{t} e(t) d t$

Burada, $k_{i}$ integral kazancidır. İkinci mertebeden bir sistem için kayma yüzeyi,

$s(t)=\dot{e}(t)+k e(t)+k_{i} \int_{0}^{t} e(t) d t$

halini alır.

Buradan, toplam kontrol sinyali,

$u=u_{e q}+u_{s w}=-f+\ddot{x}_{r}-k \dot{e}-k_{i} e-\mu \operatorname{sign}(s)$

olarak elde edilebilir. Kontrol yöntemi ile ilgili detaylı çıkarımlar Kontrol yöntemi ile ilgili detaylı çıkarımlar Kaynak 18 'de yer almaktadır.

\subsubsection{Hiyerarşik Kayan Kipli Kontrol (Hierarchical Sliding Mode Control)}

Dolaylı tahrikli ve tek girişli çok çıkışlı bir sistemin durum denklemleri aşağıdaki gibi tanımlansın.

$\dot{x}_{1}=x_{2} \& \dot{x}_{2}=f_{1}(x)+b_{1}(x) u$

$\dot{x}_{3}=x_{4} \& \dot{x}_{4}=f_{2}(x)+b_{2}(x) u$

$y(t)=\left[x_{1}, x_{2}\right]^{T}$

Burada, $x_{i}(i=1, \ldots, 4)$ sistemin durum değişkenleri, $u$ kontrol sinyali ve $y$ çıkış vektörüdür. Hiyerarşik kayan kipli kontrolde, iki ayrı grup dinamik davranış için durum değişkenleri cinsinden iki ayrı kayma yüzeyi aşağıdaki gibi tanımlanabilir [19,20,21].

$s_{1}=c_{1} x_{1}+\dot{x}_{1}$

$s_{2}=c_{2} x_{3}+\dot{x}_{3}$

Burada $c_{1}$ ve $c_{2}$ pozitif katsayılardır. Her iki denkleminde zamana göre türevleri alırsa,

$\dot{s}_{1}=c_{1} \dot{x}_{1}+\dot{x}_{2}=c_{1} x_{2}+f_{1}(x)+b_{1}(x) u$

$\dot{s}_{2}=c_{2} \dot{x}_{3}+\dot{x}_{4}=c_{2} x_{4}+f_{2}(x)+b_{2}(x) u$

Eğdeğer sinyal metodu ile alt sistemlerin eşdeğer sinyalleri,

$u_{e q 1}=-\left(f_{1}(x)+c_{1} x_{2}\right) / b_{1}(x)$

$u_{e q 2}=-\left(f_{2}(x)+c_{2} x_{4}\right) / b_{2}(x)$ 
Her bir katman için kayma yüzeyi kontrol kuralı, Lyapunov fonksiyonu ile elde edilirse, toplam kontrol sinyali,

$u=u_{2}=\left(\alpha b_{1} u_{e q 1}+b_{2} u_{e q 2}+\dot{S}_{2}\right)\left(\alpha b_{1}+b_{2}\right)^{-1}$

olarak elde edilir. Burada, $\dot{S}_{2}=-k_{2} S_{2}-\eta_{2} \operatorname{sign}_{2}$ olup $k_{2}$ ve $\eta_{2}$ pozitif katsayılardır. Lyapunov fonksiyonu $\dot{\mathrm{V}}_{2}(\mathrm{t})=\mathrm{S}_{2} \dot{\mathrm{S}}_{2}=-\eta_{2}\left|\mathrm{~S}_{2}\right|-\mathrm{k}_{2} \mathrm{~S}_{2}^{2}$ olarak seçilirse kayma yüzeyinin asimptotik kararlı olduğu görülür.

\subsubsection{Kaskat Kayan Kipli Kontrol (Cascade Sliding} Mode Control)

Kaskat kayan kipli kontrolün uygulanmasında Denklem 21'deki sistem için birinci katman kayma yüzeyi $x_{1}$ ve $x_{2}$ 'ye bağlı seçilip [23,24], katmanlara ait yüzeyler,

$s_{1}=c_{1} x_{1}+x_{2} \quad, \quad s_{2}=c_{2} x_{3}+s_{1} \quad$ ve

$s_{3}=c_{3} x_{4}+s_{2}$

olarak tanımlanır. [13,22,23].

Eşdeğer kontrol sinyali metodu ile her bir alt sistemin eşdeğer sinyali elde edilebilir.

$u_{e q 1}=f_{1}+c_{1} x_{2} / b_{1}$

$u_{e q 2}=-\left(c_{2} x_{4}+c_{1} x_{2}+f_{1}\right) / b_{1}$

$u_{e q 3}=-\left(c_{3} f_{2}\left|\operatorname{sgn}\left(s_{2}\right)\right|+c_{2} x_{4}+c_{1} x_{2}+\right.$

$\left.f_{1}\right) /\left(c_{3} b_{2}\left|\operatorname{sgn}\left(s_{2}\right)\right| b_{1}\right)$

Toplam kontrol sinyalini aşağıdaki gibi tanımlanırsa,

$u=u_{e q}+u_{s w}$

Lyapunov fonksiyonundan yararlanılarak kontrol sinyalleri,

$u_{s w}=-\left(c_{3} b_{2}+b_{1}\right)^{-1}\left[\eta \operatorname{sign}\left(s_{3}\right)+k s_{3}\right]$

$u_{e q}=\left(c_{3} b_{2}+b_{1}\right)^{-1}\left(c_{3} f_{2}+c_{2} x_{4}+c_{1} x_{2}+f_{1}\right)$

olarak elde edilir.

Lyapunov fonksiyonu $\dot{V}_{3}=-s_{3} \eta \operatorname{sign}\left(s_{3}\right)-k s_{3}^{2}$ olarak seçilirse son katman kararlılığı ve sonlu zamanda ulaşmayı garanti edebilir.

Kontrol yöntemi ile ilgili detaylı çıkarımlar Kaynak 22,23 ve 24 'te yer almaktadır.

\section{TEORİ VE DENEYSEL ÇALIŞMALARDA KULLANILAN SISTEMLER (SYSTEMS USED IN THE THEORETICAL AND EXPERIMENTAL STUDIES)}

Çalışmada karşılaştırma amacıyla kullanılan üç ayrı gerçek sistemin fiziksel modeli, matematiksel modeli ve parametreleri aşağıdaki alt başlıklarda verilmektedir.

\subsection{Basit Ters Sarkaç Sistemi (Simple Inverted Pendulum)}

Basit ters sarkaç sistemi, sabitlenmiş bir dc motor ve miline bağlı bir sarkaçtan ibarettir. Sistemin fiziksel modeli Şekil 1. parametreleri ise Tablo 1.'de görülmektedir.
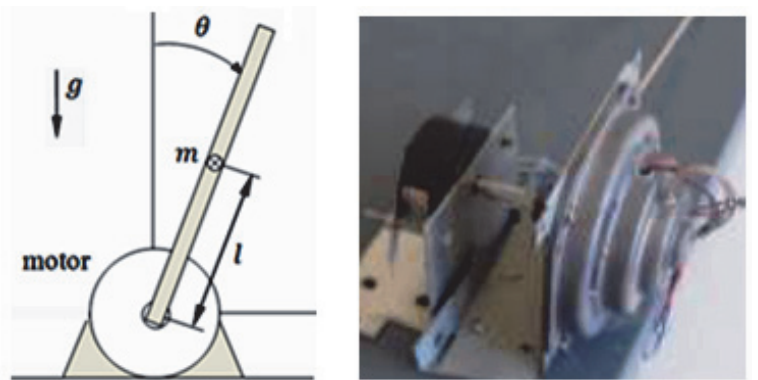

Sekil 1.Basit ters sarkacın fiziksel modeli ve resmi (Physical model and picture of the simple inverted pendulum)

Tablo 1. Basit ters sarkaç sisteminin parametreleri (Parameters of the simple inverted pendulum system)

\begin{tabular}{|c|c|c|c|}
\hline $\begin{array}{c}K_{e}=K_{t}, \text { motor tork } \\
\text { sabiti }\end{array}$ & 0.0656 & $l$, sarkacın boyu & 0.1509 \\
\hline $\begin{array}{c}R_{m}, \begin{array}{c}\text { motorelektrik } \\
\text { direnci }\end{array} \\
L_{m}, \text { motor endüktans1 }\end{array}$ & 5.1386 & $I_{m}$, atalet momenti & 0.0099 \\
\hline$M$, sarkacın kütlesi & 0.076 & $B_{t e}$, sönüm sabiti & 0.0041 \\
\hline
\end{tabular}

Dc motor tahrikli basit ters sarkacin durum denklemleri, besleme voltajı, $u$; açısal konum ve hızı $\theta=x_{1}$ ve $\omega=x_{2}$; motor bobin akımı ise $i=$ $x_{3}$ olarak tanımlanarak,

$\dot{x}_{1}=x_{2}$

$\dot{x}_{2}=\frac{K_{t}}{I_{m}} x_{3}+\frac{l m g}{I_{m}} \sin x_{1}-\frac{B}{I_{m}} x_{2}$

$\dot{x}_{3}=-\frac{K_{e}}{L_{m}} x_{2}-\frac{R_{m}}{L_{m}} x_{3}+\frac{1}{L_{m}} u$

olarak elde edilir [25].Tüm parametreler ISO birim sistemine göredir.

\subsection{De Motor Tahrikli Arabalı Sistem (De Motor Driven Cart System)}

Arabalı sistemin fiziksel modeli ve resmi Şekil 2. ve 3.' deki gibidir. 
Durum değişkenleri $x=x_{1}, v=x_{2}$ ve $i=x_{3}$ olarak tanımlanırsa, sistemin modeli,

$\dot{x}_{1}=x_{2}$

$\dot{x}_{2}=-\frac{B_{e}}{m_{e}} x_{2}+\frac{K_{t}}{m_{e} R_{g}} x_{3}$

$\dot{x}_{3}=-\frac{K_{e}}{R_{g} L_{m}} x_{2}-\frac{R_{m}}{L_{m}} x_{3}+\frac{1}{L_{m}} u$

olarak elde edilir [25]. Burada, $i$, motor akımı; $x$, konum; $u$, besleme voltajı, $v$, hızdır. Sistemin parametreleri Tablo 2.'de verilmiştir.

Tablo 2. Dc motor tahrikli arabalı sistemin parametreleri (Parameters of the dc motor driven cart system)

\begin{tabular}{|c|c|c|c|}
\hline $\begin{array}{c}K_{e}=K_{t}, \text { motor tork } \\
\text { sabiti }\end{array}$ & 0.141 & $B_{e}$, sönüm sabiti & 28.6 \\
\hline$R_{m}$, elektrik direnci & 3 & $R_{g}$, kasnak çapı & 0.027 \\
\hline$L_{m}$, motor endüktansı & 0.0159 & $m_{e}$, kütle & 1.222 \\
\hline
\end{tabular}

\subsection{Arabalı Ters Sarkaç Sistemi (Cart Type Inverted} Pendulum System)

Tek bir doğru akım servomotor ile dolaylı tahrikli iki serbestlik dereceli sistemin kinematik analizi Şekil 2.'de görülen fiziksel modelden yararlanılarak yapılmıştır[26,27].

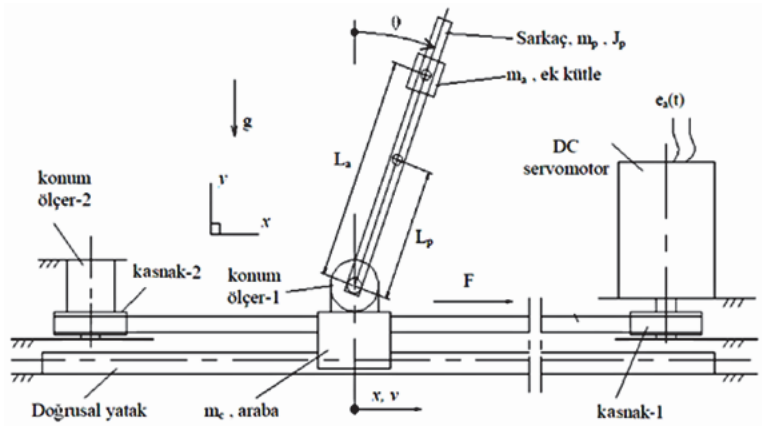

Şekil 2. Dc motor tahrikli arabalı sistem ve ters sarkaç fiziksel modeli (Physical model of the dc motor driven cart system and the inverted pendulum)

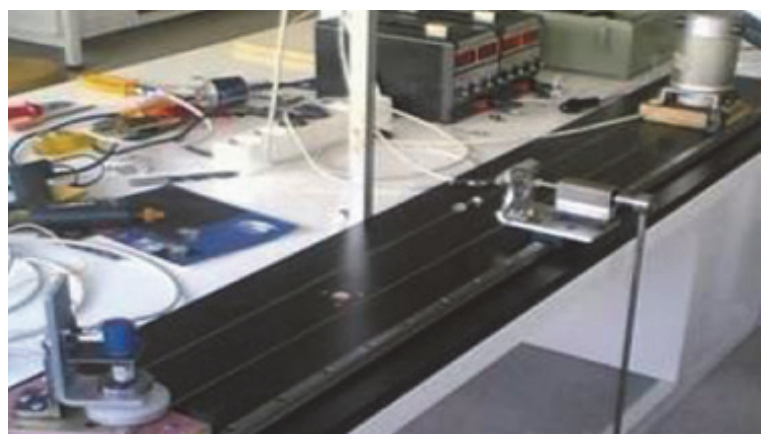

Şekil 3. Dc motor tahrikli arabalı sistem ve ters sarkacın resmi (Picture of the dc motor driven cart system)

Şekil 2.'de görülen arabalı ters sarkaç sisteminin hareket denklemleri Lagrange yöntemiyle elde edilebilir.
Lagrange denklemleri,

$\frac{d}{d t}\left(\frac{\partial L}{\partial \dot{x}}\right)-\frac{\partial L}{\partial x}=F-F_{S}$

$\frac{d}{d t}\left(\frac{\partial L}{\partial \dot{\theta}}\right)-\frac{\partial L}{\partial \theta}=M-M_{S}$

olarak yazılabilir. Sistemin durum değişkenleri,

$x_{1}=x, x_{2}=\dot{x}, x_{3}=\theta, x_{4}=\dot{\theta}$ ve $x_{5}=i_{m}$

alınarak denklemler yeniden düzenlenirse, kontrol edilecek olan sistemin durum denklemleri aşağıdaki gibi elde edilir [36].

$\dot{x}_{1}=x_{2}$

$\dot{x}_{2}=\left(\frac{c B_{m}}{R_{g} W}\right) x_{2}+\left(\frac{b^{2} g}{W} \sin x_{3} \cos x_{3}\right)$

$+\left(\frac{-b \operatorname{csin} x_{3}}{W}\right) x_{4}^{2}+\left(\frac{-c K_{t}}{R_{g} W}\right) x_{5}+\left(\frac{-b \cos x_{3}}{W}\right) M_{S}+$ $\frac{c}{W} F_{s}+\left(\frac{b \cos x_{3}}{W}\right) M_{b}(t)$

$\dot{x}_{3}=x_{4}$

$\dot{x}_{4}=\left(\frac{-b B_{m} \cos x_{3}}{R_{g} W}\right) x_{2}+\left(\frac{b^{2} \sin x_{3} \cos x_{3}}{W}\right) x_{4}^{2}$

$+\left(\frac{-a b g R_{g}^{2}-b g J_{g e}}{R_{g}^{2} W}\right) \sin x_{3}+\left(\frac{b K_{t} \cos x_{3}}{R_{g} W}\right) x_{5}$

$+\left(\frac{-b \cos x_{3}}{W}\right) F_{s}+\left(\frac{a R_{g}^{2}+J_{g e}}{R_{g}^{2} W}\right)\left[M_{s}-M_{b}(t)\right]$

$\dot{x}_{5}=\left(\frac{-K_{m}}{L_{m} R_{g}}\right) x_{2}+\left(\frac{-R_{m}}{L_{m}}\right) x_{5}+\left(\frac{1}{L_{m}}\right) e_{a}(t)$

$\mathrm{Bu}$ denklemler kontrol edilecek olan sistemin dinamiğini tanımlamakta olup, $e_{a}(t)$ sistemin kontrol girişi, $M_{b}(t)$ ise bozucu giriştir. Sistemin parametreleri Tablo 3.'de verilmiștir. Bu parametreler çeşitli yöntemlerle deneysel olarak elde edilmiştir $[28,29]$.

Tablo 3. Arabalı ters sarkacin sisteminin parametreleri (Parameters of the cart type inverted pendulum system)

\begin{tabular}{|c|c|c|c|}
\hline $\begin{array}{c}K_{e}=K_{t}, \text { motor } \\
\text { tork sabiti }\end{array}$ & 0.141 & $l_{s}$, sarkacın uzunluğu & 0.22 \\
\hline $\begin{array}{c}R_{m} \\
\text {,motorelektrik } \\
\text { direnci }\end{array}$ & 3 & $J_{s p}$, toplam atalet momenti & 0.0117 \\
\hline $\begin{array}{c}L_{m}, \text { motor } \\
\text { endüktans1 }\end{array}$ & 0.015 & $M_{s}$, kuru sürtünme & 0.0007 \\
\hline $\begin{array}{c}m_{s}, \text { sarkacin } \\
\text { kütlesi }\end{array}$ & 0.2142 & $\mathrm{~m}_{\mathrm{c}}$, araba ve kayışın kütlesi & 1.222 \\
\hline $\begin{array}{c}\mathrm{B}_{\text {te }}, \text { sönüm } \\
\text { sabiti }\end{array}$ & 28.602 & $\mathrm{~B}_{\mathrm{m}}$, motorun viskoz sönüm & 0.03 \\
\hline $\begin{array}{c}\mathrm{R}_{\mathrm{g}}, \text { çark } \\
\text { yarıçiti }\end{array}$ & 0.027 & $g$, yerçekimi ivmesi & 9.81 \\
\hline
\end{tabular}




\section{BILGISSAYAR SIMÜLASYONLARI VE DENEYSEL ÇALIŞMALAR (COMPUTER SIMULATIONS AND EXPERIMENTAL STUDIES)}

Deney düzeneği, kontrol sinyalini üreten bir bilgisayar ve yazılım (Pentium 3 ve Delphi Programlama Dili), veri iletişimini sağlayan veri toplama kartı (Advantech PCL-818HG), voltaj ve güç yükseltici devre ve sarkacın açı bilgisini ve arabanın konum bilgisini geri beslemek için direnç tipi açısal konum ölçerlerden (Gefran, doğrusallık $\mp \% 0.05$, ve 20KOhm $\mp \% 5$ doğrusallık) ve güç kaynaklarından (2 adet, 30V, 6A ayarlı ve 干5V) oluşur (Şekil 4.).

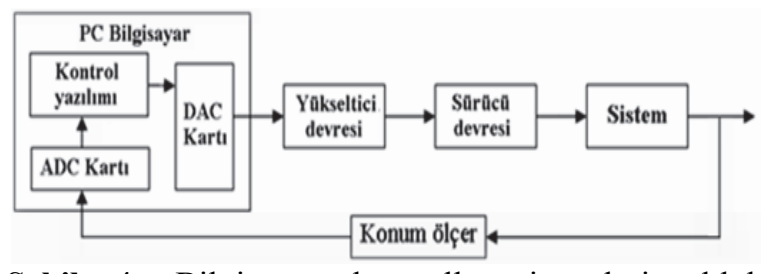

Şekil 4. Bilgisayar kontrollü sistemlerin blok diyagramı (Block diagram of the computer controlled systems)

Kontrol yazılımı Delphi görsel programlama dili kullanılarak hazırlanmıştır.

Yazılım ile veri toplama kartı üzerinden gelen sinyaller kaydedilmekte, referans girişlere göre belirlenen hatalar da dikkate alarak çalışmada kullanılan kontrol algoritmaları vasitasıyla gerekli kontrol sinyalleri üretilmektedir. Gözlemleyici kullanilan deneylerde, sistem modelinin sayisal çözümü de eklenerek kontrol sinyali elde edilmektedir.

\subsection{Basit Ters Sarkacın Kontrolü (Control of the Simple Inverted Pendulum)}

Basit ters sarkaca uygulanan çeşitli kontrol türlerinin basamak referans girişlere cevapları örnek olarak Şekil 5.'de, karşılaştırma tablosu Tablo 4.' de görülmektedir. Kontrol kazançları, simülasyon ve deneysel çalışmalarda modellenemeyen dinamik

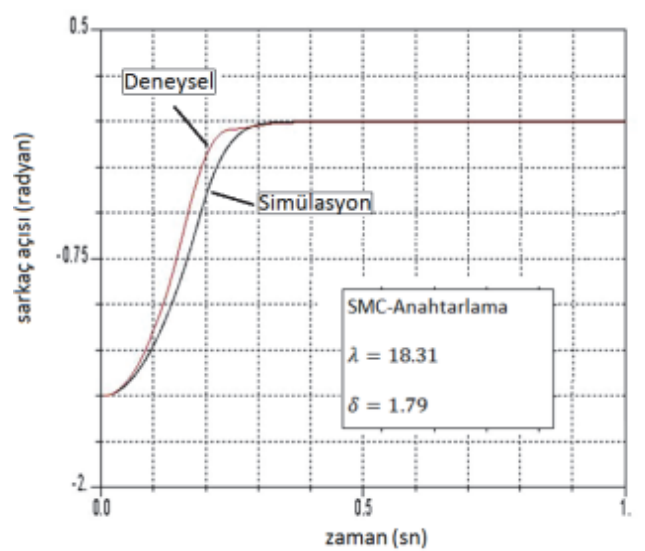

etkilerden dolayı deneme yanılma yöntemi ile belirlenmiştir. Gözlemleyici kullanılan uygulamalarda ise kök yerleştirme yöntemi ile kazançlar belirlenmiştir.

Şekillerdeki cevap eğrilerinden teorik ve deneysel olarak elde edilen sonuçlar birbirine yeterince yakın olduğu görülmektedir. Diğer yöntemler ile elde edilen sonuçlar da benzer şekilde tatmin edici düzeyde birbirine yakındır.

Tablo 4. Kontrol tekniklerinin karşılaştırma tablosu (Comparison table for the control techniques)

\begin{tabular}{|c|c|c|c|}
\hline Kontrolcü & $\begin{array}{c}\text { Așma } \\
(\mathbf{\%})\end{array}$ & $\begin{array}{c}\text { Yerleşme } \\
\text { Zamanı(sn) }\end{array}$ & $\begin{array}{c}\text { Kalıcı Hata } \\
\text { (radyan) }\end{array}$ \\
\hline PID & 0.0 & 0.44 & 0.015 \\
\hline SMC Anahtarlama & 0.0 & 0.4 & 0.006 \\
\hline SMC Toplam Sinyal & 0.0 & 0.5 & 0.005 \\
\hline ISMC Anahtarlama & 0.0 & 0.4 & 0.004 \\
\hline ISMC Toplam Sinyal & 0.0 & 0.6 & 0.002 \\
\hline SMC Gözlemleyici & 0.0 & 0.4 & 0.015 \\
\hline
\end{tabular}

Sistemde yalnızca sarkaç açısal konum bilgisi geri beslenmektedir ve hatanın dinamiğinden oluşan daha önceki yüzeylerde açısal konumun sayısal türevi alınarak hatanın türevleri elde edilmişti. Tüm durum değişkenlerinin deneysel ortamda kestirim ile elde edilebilmesi için tam mertebe Luenberger gözlemleyici tasarlanmıştır. Diğer uygulamalarda da aynı yöntem izlenmiştir.

Deneysel sonuçların 1şığında kayan kipli kontrol'ün PID kontrole göre performansının daha iyi olduğu gözlemlenmiştir. Kayan kipli kontrol ile sadece anahtarlama fonksiyonu kullanılarak elde edilen sonuçlarda, yüzey sistemin yaklaşık modeline göre seçilmiş olmasına rağmen, ulaşma zamanının ve kalıcı hatanın PID kontrol'e nazaran daha düşük olduğu gözlemlenmiştir (Tablo 4.). Yüzeye denklemine hatanın integralinin eklenmesi ile sistemin kalıcı hatasının azaldığ gözlemlenmiştir. Sistemin yaklaşık modeline göre oluşturulan bu yüzeylerde, kontrole

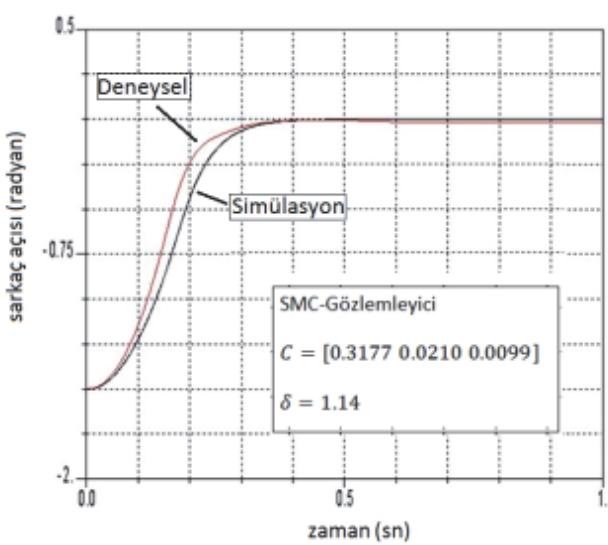

Şekil 5. Sistemin basamak referans girişlere cevapları (simülasyon ve deneysel) (System responses to step reference inputs ) (Simulation and experimental) 
eşdeğer sinyalin katılmasının sistemin yerleşme zamanını artırdığı gözlemlenmiştir. Basit ters sarkaçta modellenmeyen dinamiklerin, belirsiz doğrusallığ bozan durumların ve bozucu etkilerin az oluşu gözlemleyicinin başarısını artırmıştır.

Kök yerleştirme ile yapılan kontrolde hem anahtarlama hem de eşdeğer sinyal kullanılmış ve kontrolün iyi sonuçlar verdiği gözlemlenmiştir.

\subsection{Motor Tahrikli Arabalı Sistemin Kontrolü (Control of the DC motor driven cart system)}

DC motor tahrikli arabalı sisteme uygulanan çeşitli kontrol türlerinin teorik ve deneysel elde edilen basamak referans girişlere cevapları örnek olarak Şekil 6.-7.'de, karşılaştırma tablosu ise Tablo 5.' de görülmektedir. Kontrol kazançları, simülasyon ve deneysel çalışmalarda modellenemeyen dinamik etkilerden dolayı deneme yanılma yöntemi ile belirlenmiştir. Gözlemleyici kullanılan uygulamalarda ise kök yerleştirme yöntemi ile kazançlar belirlenmiştir.
Tablo 5. Kontrol tekniklerinin karşılaştırma tablosu (Comparison table for the control techniques)

\begin{tabular}{|c|c|c|c|}
\hline Kontrolcü & $\begin{array}{c}\text { Assma } \\
(\mathbf{\%})\end{array}$ & $\begin{array}{c}\text { Yerleşme } \\
\text { Zamanı (sn) }\end{array}$ & $\begin{array}{c}\text { Kalıcı Hata } \\
(\mathbf{c m})\end{array}$ \\
\hline PID & 8.0 & 0.34 & 0.15 \\
\hline SMC Anahtarlama & 6.1 & 0.32 & 0.05 \\
\hline SMC Toplam Sinyal & 7.2 & 0.41 & 0.07 \\
\hline ISMC Anahtarlama & 5.7 & 0.32 & 0.05 \\
\hline ISMC Toplam Sinyal & 6.0 & 0.35 & 0.12 \\
\hline SMC Gözlemleyici & 0.0 & 0.25 & 0.75 \\
\hline
\end{tabular}

Şekillerdeki cevap eğrilerinden teorik ve deneysel olarak elde edilen sonuçların birbirine yeterince yakın olduğu görülmektedir. Diğer yöntemler ile elde edilen sonuçlar da benzer şekilde tatmin edici düzeyde birbirine yakındır. Kontrolcülerin kazançları deney sırasında belirlenmiş, aşma olmaksızın sistemin yerleşme zamanını küçük tutacak kazançların seçilmesi amaçlanmış, sistemin az sönümlü olmasından dolayı yerleşme zamanını azaltmak ve referans izleme başarımını artırmak için bir miktar aşmaya müsaade edilmiştir.
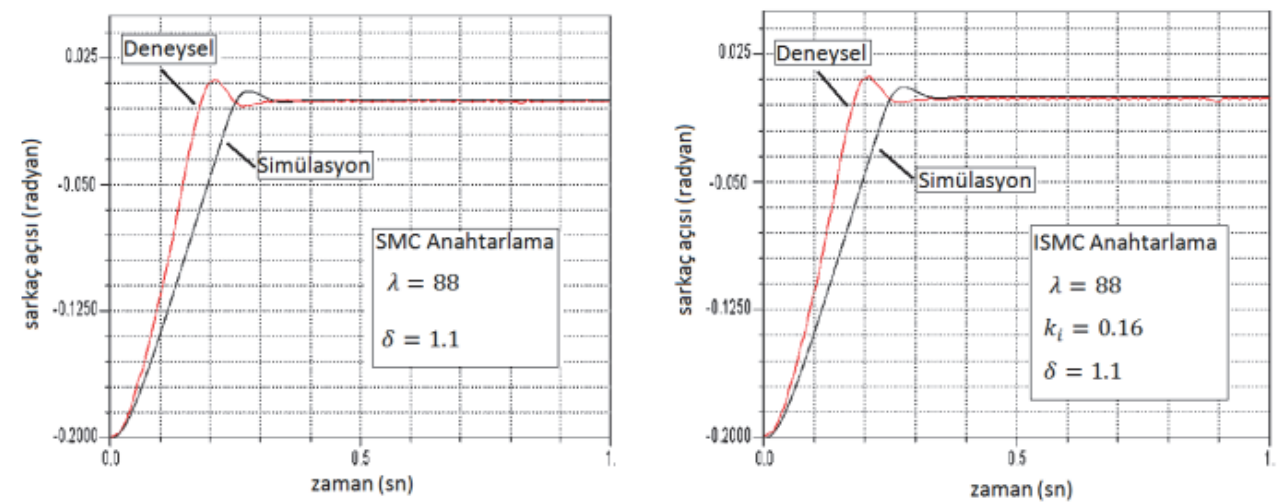

Şekil 6. Sistemin basamak referans girişlere cevapları (simülasyon ve deneysel) (System responses to step reference inputs ) (Simulation and experimental)
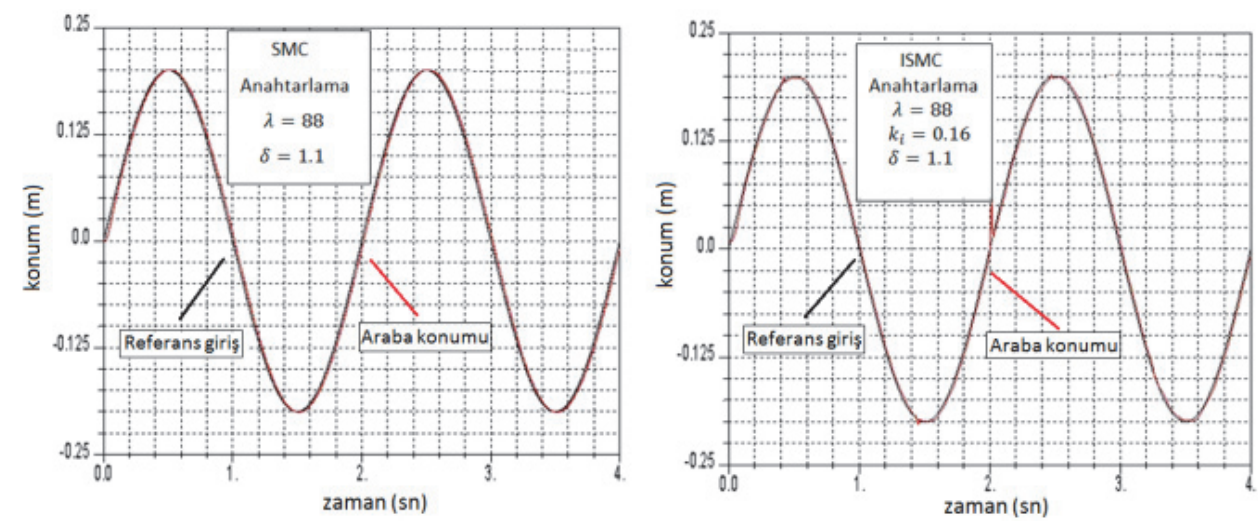

Şekil 7. Sistemin sinüs referans girişlere cevapları (Deneysel) (System responses to sinusoidal reference inputs ) ( Experimental ) 

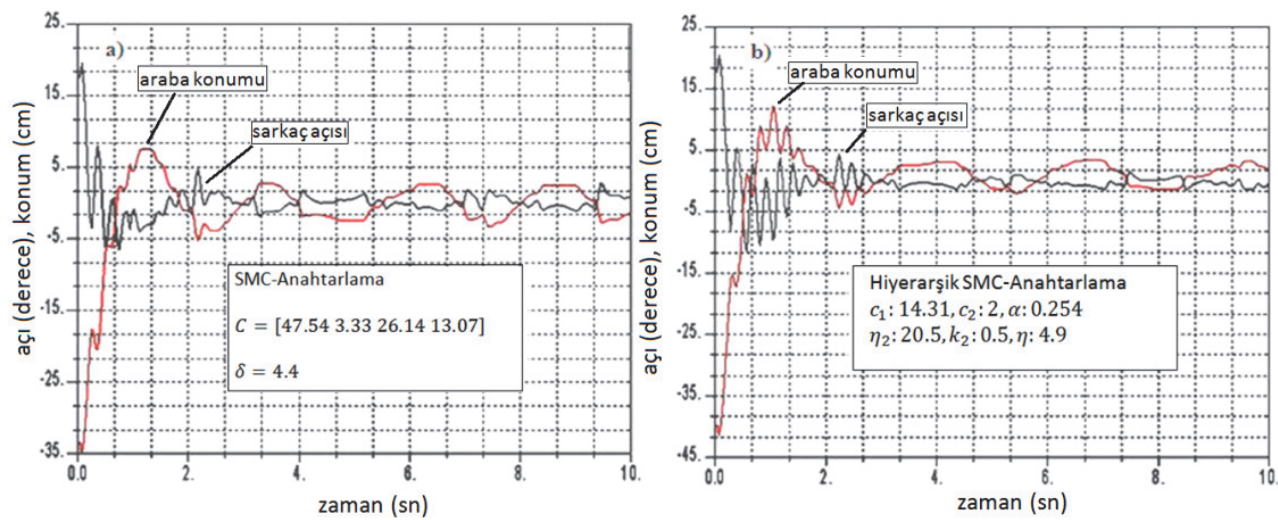

Şekil 8. Sarkaç açısı ve araba konumu zaman cevapları (Deneysel) ( System responses to step reference inputs ) (Experimental)

Gerçek sistem parametreleri ile kök yerleştirme yöntemi uygulanmış ve sistem hem simülasyonda hem de deneysel olarak aynı kontrolcü kazançları ile kontrol edilmiştir.

Dc motor tahrikli arabalı sistemde modellenmeyen dinamiklerin, doğrusallığı bozan durumların ve bozucu etkilerin basit ters sarkaç sistemine göre daha fazla olduğu gözlemlenmiştir. Bu nedenle eşdeğer sinyal ile yapılan kontrollerde anahtarlama sinyali baskın tutulmuştur. Ayrıca deney ve simülasyonlarda, deneysel olarak seçilen kontrolcü parametreleri, eşdeğer sinyalle yapılan uygulamalarda simülasyondakinden farklı seçilmiştir. Eşdeğer sinyal, sistemin eksiksiz modelini gerektirmektedir. Ancak ters sarkaçta modellenmeyen dinamiklerin, belirsiz doğrusallığı bozan durumların ve belirlenemeyen bozucu etkilerden dolayı model dinamik sistemi eksiksiz temsil edememektedir. $\mathrm{Bu}$ nedenle simülasyon için belirlenen katsayılar gerçek sistemde yeterli sonucu verememiştir. Daha eksiksiz olarak modellenebilen basit sarkaçta simülasyonda kullanılan katsayıların aynısı deneylerde de kullanılabilmiştir. Kayan kipli kontrol de eşdeğer sinyalin etkili olabilmesi için eksiksiz eşdeğer sinyalin elde edilmesinin önemi bu iki sistemden anlaşllabilmektedir. Kök yerleştirme yöntemi ile yapılan kontrolcü tasarımında hem anahtarlama hem de eşdeğer sinyal kullanılmış ancak modellenemeyen dinamikler, parametre belirsizlikleri ve bozucu etkilerden dolayı kontrolün performansının yeterli olmadığı gözlemlenmiştir.

Bilyalı doğrusal yataklama sisteminin arabası üzerine düşey olarak önemli derecede yük uygulandığında çok daha kolay kaydığı, bilya sirkülasyonunun daha hızlı gerçekleştĭgi, dolayısı ile bu yataklama sisteminin sarkaç deney düzeneği için çok uygun düşmediği, yüksüz durumda homojen olmayan davranışlar gösterdiği gözlenmiştir. Bilyalı yataklama yerine yanlardan makaralı yataklamanın daha iyi sonuçlar verebileceği düşünülmektedir.

Diğer tam modellenemeyen dinamik ise öteleme hareketini elde etmekte kullanılan çelik telin zamanla ayarı değiş̧ebilen gerginliğidir. Aynı zamanda daha hassas toleranslı parça imalatı ile deney düzeneklerinin oluşturulması gerektiği anlaşılmıştır. $\mathrm{Bu}$ durumlar dikkate alınarak karşılaştırma ve sonuç çıkarma çalışmaları yapılmış geleceğe dönük uygulama bilgi birikimi sağlanmaya çalışılmıştır.

Yukarıda bahsedilen olumsuzluklar giderildiğinde daha az motor torku yeterli olacağından, rotor atalet momenti biraz daha küçük bir motor ile aynı sonuçlar alınabilecek, hatta böylece düşük atalet momentinden dolayı daha iyi frekans cevabı ve daha kıvrak izleme özelliği elde edilmiş olacaktır.

\subsection{Arabalı Ters Sarkaç Sisteminin Kontrolü (Control of the Cart Type Inverted Pendulum System)}

Arabalı ters sarkaç sistemi doğrusal olmayan, ve tek giriş-çok çıkışlı ve dolaylı tahrikli bir sistemdir. Kontrolde amaç sistemin çıkışlarının tek kontrol sinyali ile aynı anda kontrolünün sağlanmasıdır. Sistem DC motorun dinamiği ihmal edilmeksizin modellenmiştir. Çeşitli yöntemler kullanılarak önceden elde edilmiş ve gerek görüldüğünde güncellenmiş gerçek sistem parametreler ile sistemin simülasyonu gerçekleştirilmiştir. Arabalı ters sarkacın teorik ve deneysel olarak uygulanan çeşitli kontrol türlerine cevapları örnek olarak Şekil 8.'de görülmektedir. Kontrol türlerinin karşılaştırma tablosu Tablo 6.'da verilmiştir.

Kontrol kazançları, simülasyon ve deneysel çalışmalarda modellenemeyen dinamik etkilerden dolayı deneme yanılma yöntemi ile belirlenmiştir. Sistemin kontrolünde modellemedeki belirsizliklerden dolayı anahtarlama fonksiyonları kullanılmıştır. Simülasyonlarda sarkaç açısının $\mp 0.5^{\circ}$ ve araba konumu $\mp 0.5 \mathrm{~cm}$ hassasiyetinde kontrolü gerçekleştirilmiştir.

Test edilen kontrol algoritmaları ile de aynı anda iki değişkenin kontrolü sağlanmıştır. Deneysel çalışmalarda ise sarkaç açısının $\mp 2^{\circ}$ ve araba konumu $\mp 2.0 \mathrm{~cm}$ hassasiyetinde kontrolü gerçekleştirilmiştir. 
Tablo 6. Kontrol tekniklerinin karşılaştırma tablosu (Comparison table for the control techniques)

\begin{tabular}{|c|c|c|c|c|}
\hline Kontrolcü & $\begin{array}{c}\text { Aşma } \\
\text { (Sarkaç) } \\
\text { (\%) }\end{array}$ & $\begin{array}{c}\text { Aşma } \\
\text { (Araba) } \\
\text { (\%) }\end{array}$ & $\begin{array}{c}\text { Kalıcı Hata } \\
\text { (Sarkaç)(derece) }\end{array}$ & $\begin{array}{c}\text { Kalıcı } \\
\text { Hata } \\
\text { (Araba) } \\
\text { (cm) }\end{array}$ \\
\hline SMCAnahtarlama & 34.2 & 22.6 & $\mp 2.0$ & $\mp 2.5$ \\
\hline ISMCAnahtarlama & 72.0 & 32.05 & $\mp 2.0$ & $\mp 2.5$ \\
\hline $\begin{array}{c}\text { Hiyerarşik SMC } \\
\text { Anahtarlama }\end{array}$ & 55.5 & 30.0 & $\mp 2.0$ & $\mp 2.0$ \\
\hline $\begin{array}{c}\text { Kaskat } \\
\text { SMCAnahtarlama }\end{array}$ & 90.0 & 27.7 & $\mp 2.0$ & $\mp 2.0$ \\
\hline
\end{tabular}

Araba konumu ve sarkaç açısı cevap eğrilerinde görülen düzensiz dalgalanmaların yataklama sisteminde mevcut olan tam modellenemeyen sürtünme dinamiğinden kaynaklandığı anlaşılmıştır. Bilyalı doğrusal yataklama sisteminin arabası üzerine düşey olarak önemli derecede yük uygulandığında çok daha kolay kaydığı, bilya sirkülasyonunun daha hızlı gerçekleştiği, dolayısı ile bu yataklama sisteminin sarkaç deney düzeneği için çok uygun düşmediği, yüksüz durumda homojen olmayan davranışlar gösterdiği gözlenmiştir. Bilyalı yataklama yerine yanlardan makaralı yataklamanın daha iyi sonuçlar verebileceği düşünülmektedir. Diğer tam modellenemeyen dinamik ise öteleme hareketini elde etmekte kullanılan çelik telin zamanla ayarı değişebilen gerginliğidir. Aynı zamanda daha hassas parça imalatı ve eleman seçimi ile deney düzeneklerinin oluşturulması gerektiği anlaşılmıştır. Ayrıca sistemlerdeki modellenemeyen dinamikler ve bozucu etkilerin de gözlemlenip kontrol dinamiklerine katılmasının kontrol performansını iyileştireceği düşünülmektedir.

\section{SONUÇLAR (CONCLUSIONS)}

Çalışma ile, farklı gerçek sistemlerin pratikte kullanılan bazı kayan kipli kontrol teknikleri ile kontrolleri hem teorik hem de deneysel olarak gerçekleştirilmiş, elde edilen sonuçlar değerlendirilerek kontrol teknikleri karşılaştırılmıştır.

Basit ters sarkaç sistemi modelleme hatalarının ve bozucu etkilerin az olduğu bir sistemdir. Kayma yüzeyi sistemin durum değişkenlerinden ve tam sistem modeli kullanılarak oluşturulmuş, kök yerleştirme yöntemi ile kontrolcü ve gözlemleyici tasarımlanmış, istenilen sonuçlar elde edilmiştir. Basit ters sarkaç ve doğru akım motoru tahrikli araba sisteminin ikisinde de kayan kipli kontrol hiperbolik tanjant yumuşatma fonksiyonu kullanılarak sadece anahtarlama kontrol sinyali ile gerçekleştirilmiş ve oldukça olumlu sonuçlar alınmıştır. Durum değişkenlerinin tümünü kullanarak oluşturulan kayma yüzeyi ile yapılan kontrollerde hem anahtarlama hem de eşdeğer sinyal ile kontrol gerçekleştirilmiş ve simülasyonda olumlu sonuçlar elde edilmiştir. Doğru akım motoru tahrikli arabalı sistemde modellenemeyen dinamikler ve bozucu etkilerden dolayı gözlemleyicili kontrollerde istenilen performans elde edilememiştir. Arabalı ters sarkaç sistemine anahtarlama sinyali klasik, integral, hiyerarşik ve kaskat kayan kipli kontrol teknikleri uygulanmıştır. Sistemdeki belirsizlikler, tam modellenemeyen sürtünme dinamiği ve giderilemeyen doğrusal olmayan parazitik etkilerin sistemde hassasiyet yetersizliğine neden olduğu görülmüştür. Araba konumu ve sarkaç açısı cevap eğrilerinde görülen düzensizliklerin, gürbüzlük durumundan daha çok, bir miktar zaman gecikmesinden ve hassasiyet yetersizliğinden kaynaklandığı anlaşılmıştır. Daha hassas parça imalatı ve eleman seçimi yapılarak deney düzeneğinin oluşturulması ile daha iyi kontrol sonuçları elde edilebilir.

Elde edilen sonuçlar, sistemini kayan kipli kontrol ile kontrol etmek isteyen bir uygulamacs kontrol mühendisinin hangi tür kayan kipli kontrol tekniğinin sistemine daha uygun olduğunu belirlemesine yardımcı olabilir. Yeni geliştirilen ve tutulan kayan kipli kontrol tekniklerinin eklenmesi yoluyla çalışma daha da kapsamlı hale getirilebilir.

\section{SIMGELER ( SYMBOLS )}

\begin{tabular}{|c|c|}
\hline$u_{\text {pid }}$ & : PID kontrol sinyali \\
\hline$e$ & : Sistemin hata değeri \\
\hline$K_{p}$ & : Oransal kazanç \\
\hline$T_{i}$ & : İntegral kazancı \\
\hline$T_{d}$ & : Türevsel kazanç \\
\hline$u$ & $\begin{array}{l}\text { : Kayan kipli kontrolün toplam sinyali } \\
\text { (kontrol girişi) }\end{array}$ \\
\hline$u_{s w}$ & : Kayan kipli kontrol anahtarlama sinyali \\
\hline$u_{e q i}$ & : Kayan kipli kontrol eşdeğer sinyalleri \\
\hline$T$ & : Koordinat dönüştürme matrisi \\
\hline$C$ & $\begin{array}{l}\text { : Dönüştürülmüş uzayda kayma yüzeyi } \\
\text { matrisi }\end{array}$ \\
\hline$C_{D}$ & : Durum uzayında kayma yüzeyi matrisi \\
\hline$V, V_{i}$ & : Lyapunov fonksiyonları \\
\hline$t_{e}$ & : Yüzeye ulaşma zamanı, s \\
\hline$x$ & : Sistem durum değişkeni \\
\hline$A$ & : Durum matrisi \\
\hline$B$ & : Giriş matrisi \\
\hline$A_{11}$ & : Dönüştürülmüş sistemin durum matrisi \\
\hline$A_{22}$ & : Dönüştürülmüş sistemin giriş matrisi \\
\hline$S, s_{i}$ & : Kayma yüzeyi \\
\hline$k, c_{i}$ & : Kayan kipli kontrol kazancı \\
\hline$f, f_{i}$ & $\begin{array}{l}\text { : Sistemin doğrusal olmayan durum } \\
\text { fonksiyonları }\end{array}$ \\
\hline$\alpha$ & :Yüzeyler arası ağırlık katsayısı \\
\hline$x_{r}$ & : Referans yörünge \\
\hline$b, b_{i}$ & : Sistemin kontrol girişi fonksiyonu \\
\hline$\theta$ & : Sarkacın yatayla yaptığı açı değeri, rad \\
\hline$i, i_{m}$ & : Motor bobinlerinden geçen akım, A \\
\hline$R, R_{m}$ & : Motor bobin iç direnci, Ohm \\
\hline$L, L_{m}$ & : Motor endüktans1, H \\
\hline$K_{\mathrm{e}}$ & : Motor voltaj sabiti, V/rad/s \\
\hline & : Açısal hız, rad/s \\
\hline
\end{tabular}


B : Sönüm sabiti, N.s/m

$I_{m} \quad:$ Atalet momenti, kg.m ${ }^{2}$

$l \quad:$ Sarkacın kütle merkezine kadar boyu, $\mathrm{m}$

$m, m_{s} \quad$ : Sarkaç kütleleri, kg

$K_{t} \quad:$ Motor tork sabiti, N.m/A

$R_{g} \quad$ : Kasnak yarıçapi, m

$B_{e} \quad$ : Eşdeğer viskoz sönüm sabiti, N.s/m

$m_{e} \quad$ : Eşdeğer kütle,kg

$\delta \quad:$ Sinır tabaka kalınlığ $\breve{g}_{1} \mathrm{~m}$

$M_{e l} \quad:$ Elektriksel sistemin ürettiği moment, N.m

$M_{\text {sis }} \quad$ : Ötelemeli sistemin oluşturduğu moment

$m_{c} \quad:$ Araba üzerine gelen toplam kütle, $\mathrm{kg}$

$m_{a} \quad$ : Yeri ayarlanabilen kütle, $\mathrm{kg}$

$F \quad:$ Arabaya etkiyen dış kuvvet, $\mathrm{N}$

$F_{S} \quad:$ Arabaya etkiyen kuru sürtünme kuvveti, N

$M \quad$ : Sarkaca etkiyen diş moment, N.m

$M_{s} \quad$ : Sarkaca etkiyen kuru sürtünme momenti

$B_{m} \quad$ : Motorun viskoz sönüm sabiti, N.m.s/rad

$e_{a}(t) \quad$ : Kontrol girişi, V

$M_{b}(t)$ : Bozucu moment girişi, N.m

\section{KAYNAKLAR (REFERENCES)}

1. Young, K.D., ve Utkin, V.I., and Özgüner, Ü., “A Control Engineer's Guide To Sliding Mode Control," IEEE Trans. Control Sys. Tech., Cilt 7, No 3, 328-342, 1999.

2. Utkin V.I., "Variable Structure Systems with Sliding Modes", IEEE Transactions on Automatic Control, Cilt AC-22, No 2, 212-222, 1977.

3. Utkin, V.I., "Sliding Mode Control in Mechanical Systems", IEEE Industrial Electronics, Control and Instrumentation, IECON '94, Bologna, İtalya,1429-1431, 5-9 Eylül 1994.

4. Nguyen, D., Sliding-Mode Control: Advanced Design Techniques, Doktora Tezi, University of Techology, Sydney, 1998.

5. Susperregui, A., Tapia, G. ve Y, Tapia, A., "Application of Two Alternative Sliding-Mode Control Approaches to DC Servomotor Position Tracking", IET Electric Power Applications, Cilt 1, No 4, 611-621, 2007.

6. Eker İ., "Sliding Mode Control with PID Sliding Surface and Experimental Application to an Electromechanical Plant", ISA Transactions, Cilt 45, No 1, 109-118, 2006.

7. Brandtstädter, H., ve Buss, M., "Control of Electromechanical Systems Using Sliding Mode Techniques", IEEE Conf. on Decision and Control, 44th IEEE Conference, Seville, İspanya, 1947-1952, 12-15 Aralık 2005.

8. Park, T., ve Park, K., "Sliding Mode Control Based on Pole-Placement Method for Position Control of Linear Stage", ICCAS-SICE, Fukuoka, Japonya, 5127-5130, 18-21 Ağustos 2009.

9. Nafa, F., Labiod, S. ve Chekireb, H., "A Structured Sliding Mode Controller for a Class of Underactuated Mechanical Systems", Systems,
Signal Processing and Their Applications (WOSSPA), 7th International Workshop on Systems, Tipaza, Cezayir, 243-246, 9-11 Mayıs 2011.

10. Rao, S., Brandtstädter, H., Buss, M., ve Utkin, V.I., "Sliding Mode Control in Mechanical Systems with Electric Actuators", In Proceedings of the 8th Int. Workshop on VSS, Vilanova, İspanya, 1-4, 6-8 Eylül 2004.

11. Shin, S. Y., Lee, J. Y., Sugisaka, M., ve Lee, J. J., "Decoupled Fuzzy Adaptive Sliding Mode Control For Under-Actuated Systems With Mismatched Uncertainties", Proc. IEEE Int. Conf. on Information and Automation, Harbin, Çin, 599-604, 20-23 Haziran 2010.

12. Qian, D., Yi, J., Zhao, D., ve Hao, Y., "Hierarchical Sliding Mode Control For Series Double Inverted Pendulums System", IEEE Int. Conf. on Intelligent Robots and Systems, Pekin, Çin, 4977-4982, 9-15 Ekim 2006.

13. Hao, Y.,Yi, J., Zhao, D., ve Qian, D., "Robust control Using Incremental Sliding Mode For Underactuated Systems With Mismatched Uncertainties", Proceedings of 2008 American Control Conference, Seattle, USA, 532-537, 1113 Haziran 2008.

14. Åström, K.J., Hägglund, T., PID Controllers: Theory, Design, and Tuning, Instruments Society of America, North Carolina, USA, 1995.

15. Hung, J.Y., Gao, W., ve Hung, J.C. "Variable structure control: A survey", IEEE Transactions on Industrial Elect., Cilt 40, No 1, 2-22, 1993.

16. Utkin, V.I., Sliding Modes in Control Optimization, Communication and Control, Eng. Series, Springer-Verlag, Berlin, 1992.

17. Salamc1, M.U. ve Tombul, G.S., "Sliding Mode Control Design with Time Varying Sliding Surfaces for a Class of Nonlinear Systems", IEEE Int. Conf. on Control Applications, Münih, Almanya, 996-1001, 4-6 Ekim 2006.

18. Slotine, J.J.E. ve Li, W., Applied Nonlinear Control, Prentice Hall, Englewood Cliffs, 1991.

19. Wang, W., Yi, J., Zhao, D., ve Liu, D, "Design of a Stable Sliding-Mode Controller for a Class of Second-Order Underactuated Systems", IEE Proc.-Control Theory and Applications, Cilt 151, No. 6, 683-690, Kasim 2004.

20. Qian, D., Yi, J. ve Zhao, D., "Hierarchical Sliding Mode Control to Swing up a Pendubot", Proceedings of American Control Conf. IEEE, NewYork, 5254-5259, 9-13 Temmuz 2007.

21. Qian, D., Yi, J., Zhao, D., ve Hao, Y., "Hierarchical Sliding Mode Control for Series Double Inverted Pendulums System", Int. Conf. on Intelligent Robots and Systems IEEE, Pekin, Çin, 4977-4982, 9-15 Ekim 2006.

22. Hao, Y., Yi, J., Zhao, D., ve Wang, W., "Proposal of Incremental Sliding Mode Control", Innovative Computing, Information and Cont., ICICIC'06 IEEE, Pekin, 340-343, 2006. 
23. Zehar, D., ve Benmahammed, K., "Optimal sliding mode control of the pendubot" Int. Research J. of Computer Sci. and Information Syst. (IRJCSIS), Cilt 2, No 3, 45-51, Nisan 2013.

24. Wang, W., Yi, J., Zhao, D., ve Liu, X., "Design of Cascade Fuzzy Sliding-Mode Controller", Proc. of the American Control Conf. IEEE, Portland, USA, 4649-4654, 8-10 Haziran 2005.

25. Kalayc1, M.B., Kayan Kipli Kontrol Tekniklerinin Teorik ve Deneysel Olarak İncelenmesi, Yüksek Lisans Tezi, Bozok Üniv. Fen Bilimleri Enstitüsü, 2013.

26. Yiğit, İ., "Bir Arabalı Ters Sarkaç Sisteminin Tasarımı, İmalatı ve Darbe Eni Modüleli Kayan
Kipli Kontrolü", UMTS 2007, Cumhuriyet Üniv., Sivas, Türkiye, 273-282, 7-9 Haziran, 2007.

27. Yiğit, İ., "Bir Dönel Ters Sarkaç Sisteminin Tasarımı, İmalatı ve Darbe Eni Modüleli Kayan Kipli Kontrolü”, TOK 2006 Sempozyumu, TOBB Üniv., Ankara, Türkiye, 480-487, 6-8 Kasım 2006.

28. Yiğit, İ., "Dönel Ters Sarkaç Sisteminin Modellenmesi Ve Parametrelerinin Deneysel Olarak Belirlenmesi", TOK 2010, GYTE, Gebze, Türkiye, 357-362, 21-23 Eylül 2010.

29. Yiğit, İ., "Arabalı Ters Sarkaç Sisteminin Modellenmesi ve Parametrelerinin Deneysel Olarak Belirlenmesi" ,TOK 2010, GYTE, Gebze, Türkiye, 363-368, 21-23 Eylül 2010. 